Laguillo Abbad, Clara.

Investigadora - Beca FPI, Universitat Autònoma de Barcelona, Departamento de Filosofía, grupo de investigación "Experiencia estética e investigación artística: aspectos cognitivos del arte contemporáneo" (FFI2012/32614).

\title{
La multitemporalidad en las performances del siglo XXI. Consideraciones acerca de lo simultáneo.
}

\author{
TIPO DE TRABAJO
}

Comunicación.

PALABRAS CLAVE

Directo, Diferido, Simultaneidad, Mediación, Performatividad.

KEY WORDS

Lived, Deferred, Simultaneity, Mediation, Performativity.

\section{RESUMEN}

¿De cuántas maneras vivimos el tiempo desde que tenemos tecnología a nuestro alcance? ¿Qué supone ontológicamente asistir a propuestas artísticas que combinan directo y diferido?

Tras la invención del live-feedback, vinculado al giro performativo de los 1960 que discurre en paralelo al desarrollo del vídeo y de nuevas herramientas de comunicación, podemos hablar de una tendencia hacia la hibridación de prácticas que superponen acción performativa y registro.

La combinación de registro y emisión en directo abre una noción de lo simultáneo inédita, tanto técnica como conceptualmente. Un tipo de experiencia 'multitemporal' que combina escenificación y mediación y que transmuta los problemas que se han asociado a 'lo mediado', generando cambios en el terreno de lo visible y lo perceptible.

Propuestas performativas digitales como Exmachina de Robert Lepage ponen sobre la mesa la cuestión de la experiencia multitemporal. En ellas se mezclan vídeo, sonido, performance, danza e interpretación y el espectador se ve obligado a mantener la atención en diversos estímulos y temporalidades superpuestas. La tecnología posibilita la vivencia de una narratividad múltiple del presente en vivo y el presente mediado e intervenido, que, por analogía, trasciende las fronteras entre el arte y la vida, y genera contenido y sentido por sí sola, creando una vivencia conjunta entre performers, artistas y público.

Este texto propone, pues, la revisión de la noción de simultaneidad, y pivota sobre tres ejes: la asunción de una nueva forma de mediación desde la concepción del live-feedback; el cuestionamiento desde la perspectiva tecnológica de la idea de multitemporalidad; y el reflejo de todo ello observable en ciertas producciones artísticas vinculadas a las artes performativas.

\section{ABSTRACT}

What ontological implications are conveyed by the simultaneous perception of live and recorded experiences? In how many different ways do we experience time?

After the invention of "live-feedback" in the sixties, we can speak of growing trends towards hybridization of overlapping actions and recordings. This phenomenon is one of the consequences of the performance turn in the sixties that took place simultaneously with video art and new media development. 
Overlapping recordings and live broadcasting opened up an unknown sense of simultaneity, both technical and conceptual. A kind of multitemporal experience that combines staging and mediation, and that generates changes in the visible and perceptible fields.

Performative and digital proposals found in artistic companies such as Exmachina [founded by Robert Lepage] are drawing the attention to these kind of multitemporal experiences. Their team handles video, image, sound, performance and dance, and the audience have to pay attention to multiple stimuli and different temporalities. Technology makes the experience of multiple narrativity in live present possible and the performance is similar to real life, and creates a collective action done between performers, artists and audience.

This text suggests rethinking the concept of 'simultaneity', and is structured in three main ideas: acknowledging a new way of mediation since 'live-feedback' was invented; 'multitemporal' analysis from a technological perspective; and the repercussions of both phenomena in art, especially focusing on some cross-disciplinary practices of performance art.

\section{CONTENIDO}

Esta comunicación se centra en el interés que puede suscitar la percepción del tiempo en la actualidad, en vista del nivel de tecnología que poseemos, estableciendo una relación entre la percepción temporal y la tecnología. Apunta en particular a la noción de simultaneidad analizada desde algunas prácticas performativas de la contemporaneidad. La tesis de fondo busca pensar cómo la incorporación tecnológica ha generado y genera cambios en la percepción del tiempo, y en consecuencia, también en su concepción.

La preocupación por el tiempo es tan antigua como la humanidad misma. Todos los grandes pensadores de la historia han investigado y/o cuestionado el tiempo, y a pesar de ser una inquietud compartida, el consenso se ha dado en muy escasas ocasiones. En 1984, el sociólogo Norbert Elias en su publicación Sobre el tiempo expresaba "hoy sigue sin definirse el status ontológico del tiempo. Se reflexiona sobre ello, pero no se sabe muy bien de qué tipo de objeto se trata". Entre las cuestiones clásicas relacionadas con el tiempo y la temporalidad, a menudo está el hecho de tratarse de una dimensión difícilmente asible por dinámica y cambiante. La conciencia del devenir y la finitud, o su relación con la contingencia, están entre las muchas preguntas que rodean la noción de tiempo.

Debido al hecho de que la tecnología tiene un papel fundamental en esta propuesta, será inevitable hablar de mediación. Porque a pesar de estar bien aceptado que percibimos la realidad a través de medios de diversa índole, cuando éstos implican un dispositivo tecnológico, la mediación va acompañada de una sospecha. Sospecha ante lo que nos llega, porque sus características técnicas implican que la relación con la realidad está en cierta medida intervenida, y ello puede involucrar elementos y procesos de los cuales no tenemos constancia.

Para poner en relación la percepción del tiempo en nuestra era tecnológica, con la mediación y la sospecha ante ella, se van a usar las nociones vivido y diferido. Porque asistir a algo en vivo es experimentar de forma directa en el presente y respondiendo a un ahora. $Y$ las experiencias en diferido ya son el rastro de algo pasado e irrepetible, y sólo accedemos a ellas mediante dispositivos de registro, inicialmente mecánicos, y posteriormente también digitales.

\section{Herencias: sobre el tiempo en la Modernidad}

Antes de ponernos a hablar de la percepción actual del tiempo, y en particular de la simultaneidad, conviene revisar algunas ideas sobre el tiempo propias de la Modernidad. Ello contribuirá a verificar qué hemos heredado y qué nociones son aportaciones exclusivas de nuestra era.

Entre las múltiples consecuencias de la mecanización y el desplazamiento de la atención sobre la producción propias de la Revolución Industrial, se cuenta la estandarización mundial de la hora para la regulación de los horarios del ferrocarril, el transporte marítimo y la comunicación por telégrafo. La difusión del reloj de bolsillo a finales del siglo XIX es un buen indicador de este fenómeno. Y aquí se ubica la introducción de la noción de tiempo productivo, cuya otra cara de la moneda es una nueva concepción del tiempo no productivo que se manifiesta en expresiones como tiempo perdido. El tiempo desde entonces ya no transcurre, sino que se invierte en actividades eminentemente lucrativas. ${ }^{1}$

\footnotetext{
${ }^{1}$ No cabe duda: el tiempo es oro en el XIX porque está directamente relacionado con la producción de mercancías. En el cálculo de su valor hay que tener en cuenta, además del coste de las materias primas y de las instalaciones, el salario de los obreros, que se decide en función del precio por hora trabajada. De ahí que la historia de las luchas obreras se centre, entre otras cuestiones, en torno al número de horas de trabajo a la semana
} 
La racionalización conllevó, así, una percepción de uniformidad, homogeneidad e irreversibilidad. Y en este contexto fueron las nuevas tecnologías de representación del momento como la fotografía, la fonografía o el cine, las que contribuyeron a su nueva conceptualización (DOANE, 2002).

Una de las consecuencias de esta cosificación moderna del tiempo es su consideración como fragmentación en unidades, planteada mediante la noción de instante, y que llevó implícita una obsesión por su definición y representación. Una prueba de ello es el interés renovado por las Paradojas de Zenón, o, en el otro lado de la misma cuestión, las cronofotografías de Étienne-Jules Marey y los experimentos fotográficos de Eadweard Muybridge.

En términos materialistas, esta obsesión por la cuantificación del tiempo y su reducción a divisiones estáticas puede ser leída como un retorno al atomismo, donde la definición del tiempo pasaría por el análisis de su tamaño, su forma y hasta su número.

¿Fue la aparición de medios de representación mecánica como la fotografía, la fonografía o el cine, detonante de esta concepción de fragmentación del tiempo? ¿O el desarrollo de estas tecnologías posibilitó una representación del mismo que en cierta medida legitimaba este intento de definición del instante? Porque, de alguna manera, la idea de instante quedaba perpetrada por el espejismo de tiempo detenido que posibilitaba el disparo fotográfico.

Sea como fuere, ello entraba en contradicción con una idea previa con la que convivió durante la Modernidad: el tiempo es un continuo indivisible.

Porque efectivamente resultaba y resulta difícil concebir realmente la divisibilidad e inmovilidad vinculada a la noción de instante, de la Modernidad hemos heredado también la concepción del tiempo como dinamismo genuino, que admite de una forma más natural la idea de simultaneidad.

Así, lo cierto es que hoy bebemos de una doble concepción temporal que, a pesar de venir gestándose en los periodos anteriores, se fue definiendo de una forma más evidente tras las implicaciones socio-culturales y económicas que trajo la Revolución Industrial y, finalmente, se acabó afianzando en la Modernidad. Así pues, de un lado el tiempo como una consecución lineal de instantes; y del otro, el tiempo como un continuo donde se producen superposiciones permanentemente. Esta disyuntiva entre continuidad y discontinuidad se manifestó en las reflexiones sobre el continuo de Peirce, sobre las de Bergson acerca de la duración, se relaciona con la memoria, según Freud, está presente en Proust y Joyce, y también en el concepto de schock de Benjamin, por poner unos cuantos ejemplos. En todas ellas, el punto de partida era una necesidad de visualización del tiempo, y por lo tanto, una vinculación con su representabilidad.

La tesis que sostengo en esta comunicación, parte de la presunción de que la tecnología del momento, y en concreto la fotografía y el cine, jugaron un papel crucial en esta doble concepción. Y mi contribución más esencial en este punto es que, de los dos medios que mejor desarrollaron el propósito de representar el tiempo, cada uno asistió una de las dos visiones; esto es, la fotografía alimentó la idea del tiempo lineal basado en la consecución de instantes; y el cine, la de la concepción del tiempo como un continuo de superposiciones.

La idea de simultaneidad está, desde mi punto de vista, presente en ambas formas de acceso a la temporalidad. En el caso del instante, por una conciencia de que éste puede acoger diversas experiencias al mismo tiempo; y en el del continuo, porque sin abstracciones divisionales, la conciencia acepta mejor la idea de una experiencia múltiple.

Siguiendo esta lógica, si en parte el concepto de simultaneidad es diferente hoy, lo es por la hibridación en la práctica artística de esta doble noción moderna del tiempo que hemos heredado: porque cuando la foto, vinculada al instante, y el vídeo, heredero del cine y asociado con la duración, están en combinación, generan experiencias de simultaneidad con un mayor grado de complejidad.

\section{Hibridación y multitemporalidad}

La hibridación de medios es, pues, una pieza clave en el análisis de la percepción contemporánea del tiempo contemporáneo si se piensa desde la noción de simultaneidad que a su vez se apoya en la conciencia de la mediación tecnológica. Y parte del sentido lo adquiere cuando se piensa en relación a dos fenómenos propios de los años sesenta y setenta del siglo XX de los cuales no puede disociarse: de un lado, el giro performativo en las artes, y del otro, la invención y el uso del live-feedback en prácticas artísticas.

En los años sesenta la performance entra en la escena artística de forma definitiva y ello conlleva el desplazamiento del interés del objeto al proceso de creación. En paralelo discurre el desarrollo del videoarte, hecho que implica una convivencia y una retroalimentación de la performance hacia el vídeo y viceversa desde los orígenes de ambas disciplinas. A pesar de ello, la dos prácticas encarnan esa dupla aparentemente antagónica planteada al inicio: la de las experiencias en vivo y las experiencias en diferido.

Las implicaciones filosóficas de la entrada en escena de la performance en el arte deben leerse desde la fascinación por la contingencia y lo efímero y la contingencia, también heredada también de la Modernidad, y que, ante la existencia y el desarrollo de un medio de 
registro cinético y ágil como el vídeo, ilumina una tensión epistemológica entre una urgencia de inmediatez y una necesidad de permanencia. Tensión que ha ido in crescendo desde entonces hasta hoy.

Pero, como ya se ha planteado al inicio, la mediación asociada a dispositivos mecánicos y/o digitales como la fotografía y el vídeo, va acompañada de una sospecha, la de la posibilidad del engaño, que forma parte, parcialmente, de una lenta aceptación de la limitación del cuerpo humano y sus sentidos. Esta sensación de engaño se alimenta también de las investigaciones sobre lo instantáneo más arriba mencionadas (Muybridge, Marey, etc) porque, si se acepta su existencia de alguna manera se asumen pérdidas perceptivas entre un instante y el siguiente.

Por ese motivo resulta fundamental en este contexto la invención del live-feedback a finales de los sesenta, a partir de la cual la combinación de registro y emisión en directo desarticula la presunción de engaño, transmutando los problemas asociados a la mediación, y abriendo una noción de lo simultáneo inédita, tanto técnica como conceptualmente. Un acceso a lo diferido en tiempo real que genera cambios y ampliaciones en el terreno de lo visible y lo perceptible, y desplaza el foco de nuevo hacia la experiencia vivida y, sin embargo, mediada.

Y en la tendencia hacia la hibridación de prácticas que superponen acción performativa, registro y emisión en directo que se dio a partir de los sesenta, una de las piezas que resulta paradigmática es la titulada Live/Taped Corridor (1970) ${ }^{2}$ de Bruce Nauman, donde el artista alude a diferentes temporalidades: por un lado, el tiempo vivido y el diferido, por el otro, lo simultáneo, lo mediado, lo real y hasta lo (proto)virtual. Y ofrece, así, un tipo de experiencia que voy a llamar a partir de ahora experiencia multitemporal.

En el momento de concepción de esta obra, considerada también performativa, Nauman estaba interesado por los límites biológicos del organismo humano y tenía especial interés en lo mecánico. En este contexto, en Live/Taped Corridor el artista desaparece y deja el lugar de construcción/creación de la pieza a los asistentes a la misma ${ }^{3}$. En su recorrido por la videoinstalación, éstos viven el espacio en presente, y también desde la mediación, gracias a las cámaras en sistema live-feedback ubicadas en uno de los extremos del pasillo, al inicio del mismo. El primero de los dos monitores situados al final de la misma retransmite una imagen del pasillo vacío, simulando un tiempo irreal, por detenido, diferido y, por lo tanto, sospechoso; el segundo, retransmite en directo el recorrido de los asistentes, pero como la cámara se halla al inicio del recorrido, la retransmisión tiene algo de alienante, pues los propios visitantes se alejan de sí mismos a medida que se acercan a los monitores.

Esta propuesta de Nauman resulta paradigmática porque es una instalación (proto)digital interactiva, en la que la tecnología del livefeedback genera en los asistentes una experiencia que combina múltiples temporalidades simultáneamente, tanto reales como mediadas, sin por ello dejar de cuestionar, por un lado el dispositivo de registro, y por el otro, los límites de la biología humana.

En este punto probablemente sea necesario preguntarse ¿qué supone ontológicamente percibir lo multitemporal? ¿Y con qué grado de naturalidad tenemos hoy incorporado este tipo de experiencia hoy?

Aunque realmente no existe una respuesta concreta a estas dos cuestiones, sí se pueden plantear algunas reflexiones en torno a ellas. De entrada, percibir lo multitemporal implicaría experimentar diversas temporalidades simultáneamente, como las sugeridas en la pieza escogida de Nauman.

El concepto de simultaneidad de alguna manera lleva implícita la idea de instante, puesto que para que algo ocurra a la vez, implica que está inscrito en un lapso temporal, y éste debe ser al menos cuestionado o aceptado como tal.

Si partimos de su aceptación, es un lugar común que la idea de instante no puede desvincularse del momento histórico en el que se problematiza. Ello explica que antes de la Revolución Industrial el tiempo no transcurriera de la misma manera a nivel perceptivo. $\mathrm{O}$ que nuestros relojes actuales, asociados a nuestros teléfonos inteligentes, digitales y sincronizados vía satélite con el resto del planeta, nos permitan dar la hora con un nivel de exactitud inconcebible hace tan solo un cuarto de siglo, cuando nuestros relojes de pulsera daban una hora bastante exacta, pero fluctuante en minutos. Por lo tanto, aceptamos que la percepción del concepto de instante en nuestro caso se relaciona con la tecnología en algún sentido, que a su vez nos proporciona la mayor velocidad alcanzada jamás en distancias, procesos y transmisión de la información. Porque la realidad es que no vivimos sin tecnología en ninguno de los ámbitos de nuestra existencia.

Dicho esto, y sin la necesidad de definir qué es exactamente un instante hoy, una experiencia multitemporal inscrita en él, sea de la naturaleza que sea, no existe sin alguna forma de intervención o mediación tecnológica.

\footnotetext{
${ }^{2}$ Fecha de la primera versión, porque la instalación fue repetida en numerosas ocasiones a partir de 1970

${ }^{3}$ He puesto cuidado en la elección de esta palabra, "asistente", porque son ellos quienes hacen la pieza, en la medida en que la completan. Las nociones de espectador y visitante no valen ya
} 
En consecuencia, la propuesta es aceptar que vivimos a diario experiencias multitemporales, y plantearlo como una tendencia hacia una mayor capacidad de atención de diferentes estímulos simultáneamente. En caso de que dicha tendencia pueda verificarse, desde mi punto de vista surge la siguiente pregunta: ¿en qué medida esta asimilación progresiva de multiestímulos, asistida por la mediación tecnológica, puede llegar a inscribirse en nuestro ADN cultural, es decir, el adquirido? Y la pregunta queda lanzada, sin encontrar respuesta aún, porque plantearla implica pensar en la modificación de acceso al conocimiento, que, más allá de la reflexión filosófica, tendría también implicaciones pragmáticas.

Y queda de lado, de momento, la revisión exhaustiva de los elementos que diferenciarían las experiencias multitemporales artísticas y vitales, que es en definitiva la revisión de una pregunta clásica en el arte: la de su vinculación o desvinculación con la vida, aunque en parte ya se haya dado una respuesta.

\section{Performatividad en el siglo XXI}

Volviendo de nuevo al terreno definido por la simultaneidad, la multitemporalidad, la tecnología y el arte, las prácticas performativas de nuestro siglo son las representantes artísticas idóneas para pensar en todo ello, porque se trata de propuestas que en sí mismas se construyen en torno a la temporalidad.

¿Son diferentes respecto a las performances de los inicios? En esencia, no hay diferencias estructurales entre las performances de hoy y las de los sesenta y setenta. Como ya ocurría entonces, tienden de forma natural a la hibridación y, por extensión, fagocitan en muchas ocasiones la mediación tecnológica, en parte porque como propuestas evolucionaron en paralelo e influenciándose permanentemente entre ellas. Pero resulta estéril hablar de una forma tan general de una práctica que se define por su amplísima casuística, de manera que en este último tramo la atención se centra en algunas compañías de teatro concretas que, desde finales de los noventa y hasta hoy, realizan producciones que ya no son solo dramáticas, sino que trabajan a base de híbridos performativos y tecnológicos, generando interesantes experiencias en vivo y en diferido, de simultaneidad y multitemporalidad.

La compañía Exmachina fundada en el año 1994 en Francia por el dramaturgo, actor y escenógrafo Robert Lepage (Québec, 1957), realiza producciones que mezclan vídeo, sonido, performance, danza e interpretación, además de escenografías técnicamente complejas. Sus propuestas obligan al espectador a mantener la atención en diversos lugares espaciales, mezclando temporalidades. La audiencia vive el directo y el diferido simultáneamente, y la tecnología posibilita vaivenes en el tiempo y en el espacio, y genera sentido y contenido por sí sola. El espacio escénico se convierte en el lugar de la experiencia compartida, de una doble narratividad del presente en vivo y el presente mediado, que, por analogía, trasciende las fronteras entre el arte y la vida.

En Zulu Time (1999), por ejemplo, el público vivía un frenesí de sonidos sintetizados, destellos de luz, mezcla de vídeo y la aparición de acróbatas y contorsionistas, que explicaba la historia de personajes que comparten la noción de trayecto, tránsito, cambio de estatus y transformación. Y se escenificaba todo en un entorno tecnológico que posibilitaba la significación de varias percepciones de la realidad al mismo tiempo. Una pieza sobre la ubicuidad, que hacía uso de diversos medios de registro, manipulación y reproducción en combinación con experiencias en directo.

Propuestas similares son las de la Fura dels Baus, donde el fenómeno escénico y los experimentos tecnológicos desdibujan las fronteras entre disciplinas, generando experiencias de una realidad extendida a través de la virtualidad derivada de la mediación tecnológica.

También está el trabajo de la Agrupación Señor Serrano, fundada en 2006 por Álex Serrano, donde el escenario deviene laboratorio de interacción, y se tematiza la relación con el tiempo -en contenido y forma- mediante propuestas articuladas a partir de proyecciones a gran escala de sucesiones de acciones basadas en la manipulación en tiempo real, registradas, y transformadas en la realidad y en la virtualidad.

En conclusión, tres colectivos que exploran los mecanismos de la percepción a través de una hibridación de medios con lenguajes performativos, y que definen hoy experiencias estéticas, múltiples y simultáneas, en las que, como vivencias de la temporalidad, la tecnología se revela constituyente y constitutiva de la obra de arte. Un tipo de experiencias que, desde mi punto de vista, abre las puertas de la multitemporalidad, y que definen, a través de lo vivido y lo diferido, una parte de nuestras formas de acceso a y comprensión de la realidad. 


\section{FUENTES REFERENCIALES.}

. CROSBY, Alfred W. La medida de la realidad. La cuantificación y la sociedad occidental 1250 - 1600. Crítica, 1998.

- DOANE, Mary-Ann: La emergencia del tiempo cinemático. Modernidad, contingencia y archivo. CENDEAC, Murcia, 2012.

- ELIAS, Norbert: Sobre el tiempo. FCE, Madrid, 1989.

· FLUSSER, Vilém: Hacia una filosofía de la fotografía. Editorial Trillas, México, 1990.

- GROOM, Amelia (Ed.): TIME. White Chapel Gallery-The MIT Press, Colección Documents of Contemporary Art, Londres, 2013.

· SCHULTZ, Margarita: Filosofía y producciones digitales. Alfagrama, Buenos Aires, 2006. 